\title{
Downregulation of miR-10b promotes osteoblast differentiation through targeting Bcl6
}

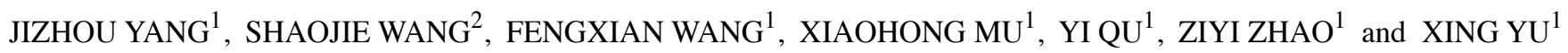 \\ ${ }^{1}$ Department of Orthopaedics, Dongzhimen Hospital Affiliated to Beijing University of Chinese Medicine, \\ Beijing 100700; ${ }^{2}$ Department of Orthopaedics, Dongfang Hospital, \\ Beijing University of Chinese Medicine, Beijing 100078, P.R. China
}

Received November 16, 2016; Accepted April 5, 2017

DOI: 10.3892/ijmm.2017.2955

\begin{abstract}
MicroRNAs (miRNAs or miRs) have been shown to play a critical role in osteoblast differentiation. miR-10b has been found to be downregulated during osteoblast differentiation; however, its precise effect on osteoblast differentiation remains unknown. In this study, we aimed to investigate the potential role of miR-10b and the potential underlying mechanism in regulating osteoblast differentiation. We found that miR-10b was downregulated during osteoblast differentiation. Overexpression of miR-10b inhibited osteoblast differentiation, whereas the suppression of miR-10b promoted osteoblast differentiation. Bioinformatics analysis and the dual-luciferase reporter assay demonstrated that miR-10b could target the 3 '-untranslated regions of $\mathrm{B}$ cell lymphoma $6(\mathrm{Bcl} 6)$ which is an important regulator of osteoblast differentiation. Real-time quantitative polymerase chain reaction and western blot analysis showed that miR-10b directly regulated Bcl6 expression. Further experiments showed that the overexpression of miR-10b increased the expression of signal transducer and activator of transcription 1 (STAT1) and blocked Runt-related transcription factor 2 (Runx2) nuclear translocation, whereas miR-10b suppression showed an opposite effect. Moreover, the miR-10b suppression-induced effects were partially reversed by Bcl6 knockdown. Taken together, our study suggests that miR-10b contributes to osteoblast differentiation through targeting Bcl6, providing a novel insight into understanding
\end{abstract}

Correspondence to: Dr Shaojie Wang, Department of Orthopaedics, Dongfang Hospital, Beijing University of Chinese Medicine, 6 Fangxingyuan, Fengtai, Beijing 100078, P.R. China E-mail: shaojiewang64@163.com

Abbreviations: miRNAs, microRNAs; UTR, untranslated regions; Bcl6, B cell lymphoma 6; Runx2, Runt-related transcription factor 2; STAT1, signal transducer and activator of transcription 1; RT-qPCR, real-time quantitative polymerase chain reaction; ALP, alkaline phosphatase; OPN, osteopontin; COL1A1, collagen type I $\alpha 1$

Key words: osteoblast differentiation, miR-10b, Bcl6, signal transducer and activator of transcription 1 the molecular mechanism underlying osteoblast differentiation and suggesting a potential target for inhibiting bone loss.

\section{Introduction}

Bone homeostasis is controlled by the balance between resorption and bone formation, mediated by osteoblasts and osteoclasts $(1,2)$. The excessive bone loss results in osteoporosis which is a common disease among women worldwide after menopause (3). However, current treatments for bone mass recovery are still limited. Osteoblasts are a specialized subset of cells that play an important role in bone formation (1). Osteoblasts originate from mesenchymal stem cells that secrete alkaline phosphatase (ALP) and bone matrix proteins, including osteopontin (OPN) and collagen type I $\alpha 1$ (COL1A1) (4). Targeting osteoblast differentiation has become a promising therapeutic strategy for osteoporosis.

Osteoblast differentiation is mediated by a variety of multiple factors, including microRNAs (miRNAs or miRs) (5). miRNAs are a group of small RNAs, 22 nucleotides in length, which negatively regulate gene expression by targeting the 3'-untranslated regions (3'-UTR) (6-8). By post-transcriptionally modulating gene expression, miRNAs can regulate numerous biological processes, including cell proliferation, apoptosis and differentiation (6-8). In recent years, a growing body of evidence has reported that miRNAs play a critical role in regulating osteoblast differentiation (9-12). Therefore, targeting osteoblast differentiation by miRNAs may show great promise for the treatment of bone loss diseases, such as osteoporosis.

Runt-related transcription factor 2 (Runx2) is a master transcription factor for controlling osteoblast differentiation (13-16). The signal transducer and activator of transcription 1 (STAT1) has been found to be a critical regulator for Runx2 (17). STAT1 can interact with Runx2 and thus restrain Runx 2 in the cytoplasm leading to the inhibition of osteoblast differentiation (18). STAT1 has been shown to promote bone resorption in mice $(19,20)$. Interestingly, B cell lymphoma 6 (Bcl6) has been reported as a transcriptional repressor for STAT1 (21). Bcl6 is primarily expressed in B lymphocytes and plays an important role in regulating $B$ lymphocyte growth and development $(22,23)$. It has been found that Bcl6 inhibits the expression of STAT1 
and thus promotes osteoblast differentiation (21). Therefore, Bcl6/STAT1/Runx2 signaling plays an important role in bone homeostasis targeting which may provide a novel strategy for the control of osteoblast differentiation.

miR-10b has been suggested as a regulator for cell differentiation $(24,25)$. A recent study has reported that miR-10b shows decreased expression during osteoblast differentiation (26). However, the precise effect of miR-10b on osteoblast differentiation remains unknown. In this study, we aimed to investigate the potential role of $\mathrm{miR}-10 \mathrm{~b}$ and the potential underlying mechanism in regulating osteoblast differentiation. We found that miR-10b was downregulated during osteoblast differentiation. Overexpression of miR-10b inhibited osteoblast differentiation, whereas suppression of miR-10b promoted osteoblast differentiation. Bcl6 was identified as a target gene of miR-10b in osteoblast differentiation. miR-10b regulated Bcl6 expression as well as STAT1/Runx2 signaling. However, the miR-10b suppression-induced effects were partially reversed by Bcl6 knockdown. Taken together, our study suggests that miR-10b contributes to osteoblast differentiation through targeting Bcl6, providing novel insight into understanding the molecular mechanism underlying osteoblast differentiation and suggesting a potential target for inhibiting bone loss.

\section{Materials and methods}

Cell culture. Pre-osteoblast MC3T3-E1 cells were purchased from the Type Culture Collection of the Chinese Academy of Sciences (Shanghai, China) and cultured in Alpha Modified Eagle's Medium ( $\alpha$-MEM; Invitrogen, Carlsbad, CA, USA) containing $10 \%$ fetal bovine serum (FBS; Gibco, Rockville, MD, USA) and 1\% penicillin/streptomycin (Sigma-Aldrich, St. Louis, MO, USA). The cells were grown in a humidified atmosphere of $5 \% \mathrm{CO}_{2}$ at $37^{\circ} \mathrm{C}$. To induce osteoblast differentiation, cells were grown in osteogenic differentiation medium (HyClone, Logan, UT, USA) supplemented with 10\% FBS, $50 \mu \mathrm{g} / \mathrm{ml}$ ascorbic acid and $10 \mathrm{mM}$ sodium $\beta$-glycerophosphate. The medium was refreshed every two days for the induction of osteoblast differentiation (27).

Quantitative (real-time) polymerase chain reaction ( $R T-q P C R)$. Total RNAs or miRNAs were extracted by TRIzol (Invitrogen) or mirVana miRNA isolation kit (Applied Biosystems, Foster City, CA, USA), respectively. For mRNA detection, total RNAs were reverse-transcribed into cDNA by M-MLV reverse transcriptase (Takara, Dalian, China). For miRNA detection, miRNAs were reverse-transcribed into cDNA by the TaqMan microRNA reverse transcription kit (Applied Biosystems). qPCR was performed using Power SYBR-Green PCR Master Mix on an Applied Biosystems AB7500 Real-Time PCR system (both from Applied Biosystems) following the procedures: $94^{\circ} \mathrm{C}$ for $5 \mathrm{~min}, 30$ cycles of two-step cycling program $\left(94^{\circ} \mathrm{C}\right.$ for $10 \mathrm{sec}, 60^{\circ} \mathrm{C}$ for $20 \mathrm{sec}$ and $72^{\circ} \mathrm{C}$ for $30 \mathrm{sec}$ ), and $72^{\circ} \mathrm{C}$ for $10 \mathrm{~min}$. Glyceraldehyde 3-phosphate dehydrogenase (GAPDH) and small nuclear RNA U6 served as the internal controls. Relative gene expression was quantified by using the $2^{-\Delta \Delta \mathrm{Ct}}$ method. The primer sequences were as follows: miR-10b forward, 5'-TACCCTGTAGAACCGAATTTG-3' and reverse, 3'-GTGCGTGTCGTGGAGTC-5'; U6 forward, 5'-CGCTTCAC GAATTTGCGT-3' and reverse, 5'-CTCGCTTCG CAGCA
CA-3'; Bcl6 forward, 5'-AGACGCACAGTGACAAACCAT ACA-3' and reverse, 5'-CTCCACAAATGTTACAGCGAT AGG-3'; ALP forward, 5'-CACCATTTTTAGTACTGGCCA TCG-3' and reverse, 5'-GCTACATTGGTGTTGAGCTTTGG-3'; OPN forward, 5'-TCTCCTTGCGCCACAGAATG-3' and reverse, 5'-TCCTTAGACTCACCGCTCTT-3'; COL1A1 forward, 5'-CCCCGGTCAGAGAGGAGAAA-3' and reverse, 5'-TCC AGAAGGACCTTGTTTGC-3'; GAPDH forward, 5'-AATGG A TTTGGACGCATTGGT-3' and reverse, 5'-TTTGCACTGGTA CGTGTTGAT-3'.

Transfection. The miR-10b mimics, miR-10b inhibitor and negative control (NC) were purchased from GenePharma (Shanghai, China) and transfected into cells using Lipofectamine 2000 (Invitrogen) according to the manufacturer's protocols. Bcl6 siRNA and NC siRNA were obtained from Santa Cruz Biotechnology, Inc. (Santa Cruz, CA, USA) and transfected into cells as per the recommended methods. The transfection efficiency was evaluated by RT-qPCR or western blot analysis.

ALP activity assay. Cells were transfected with miR-10b mimics or miR-10b inhibitor followed by the induction of osteoblast differentiation for 6 days. Then, the cells were harvested and detected by ALP assay kit. Briefly, the cells were lysed in lysis buffer and the supernatants were collected and incubated with SensoLyte $p$-nitrophenylphosphate at $37^{\circ} \mathrm{C}$ for $30 \mathrm{~min}$. The absorbance at a wavelength of $405 \mathrm{~nm}$ was detected by an enzyme-linked immunosorbent assay (ELISA) reader (Bio-Rad, Hercules, CA, USA).

Measurement of matrix mineralization. After the induction of osteoblast differentiation, cells were harvested and fixed in $70 \%$ ethanol for $1 \mathrm{~h}$. Then, cells were incubated with $40 \mathrm{mM}$ Alizarin Red S solution (Sigma-Aldrich) for $10 \mathrm{~min}$. The mineral deposits stained by Alizarin Red S were isolated and dissolved in $0.1 \mathrm{~N} \mathrm{NaOH}$. The absorbance at a wavelength of $540 \mathrm{~nm}$ was measured by an ELISA reader (Bio-Rad).

Dual-luciferase reporter assay. Bioinformatics analysis was performed by using microRNA.org-Targets and Expression (http://www.microrna.org/) and TargetScan (http://www. targetscan.org/). The miR-10b target region of Bcl63'-UTR was inserted into a pmirGLO luciferase vector (Promega, Madison, WI, USA) to obtain wild-type pmirGLO-Bcl6 3'-UTR. Meanwhile, Bcl6 3'-UTR sequences containing the mutant binding sites for miR-10b were cloned into a pmirGLO luciferase vector (Promega) to obtain mutant-type pmirGLO-Bcl6 3'-UTR. To confirm the interaction between miR-10b and Bcl6 3'-UTR, wild-type or mutant-type pmirGLO-Bcl6 3'-UTR was co-transfected into MC3T3-E1 cells with miR-10b mimics or miR-10b inhibitors. After incubation for $48 \mathrm{~h}$, the cells were harvested and the relative luciferase activity was measured by Dual-GLO Luciferase assay system (Promega).

Western blot analysis. Cytosolic and nuclear fractions were extracted using the nuclear extraction kit (Beyotime, Haimen, China) according to the manufacturer's protocols. Briefly, cells were harvested and washed with phosphate-buffered saline (PBS) followed by centrifugation at $8,000 \mathrm{x} \mathrm{g}$ for $15 \mathrm{~min}$ 

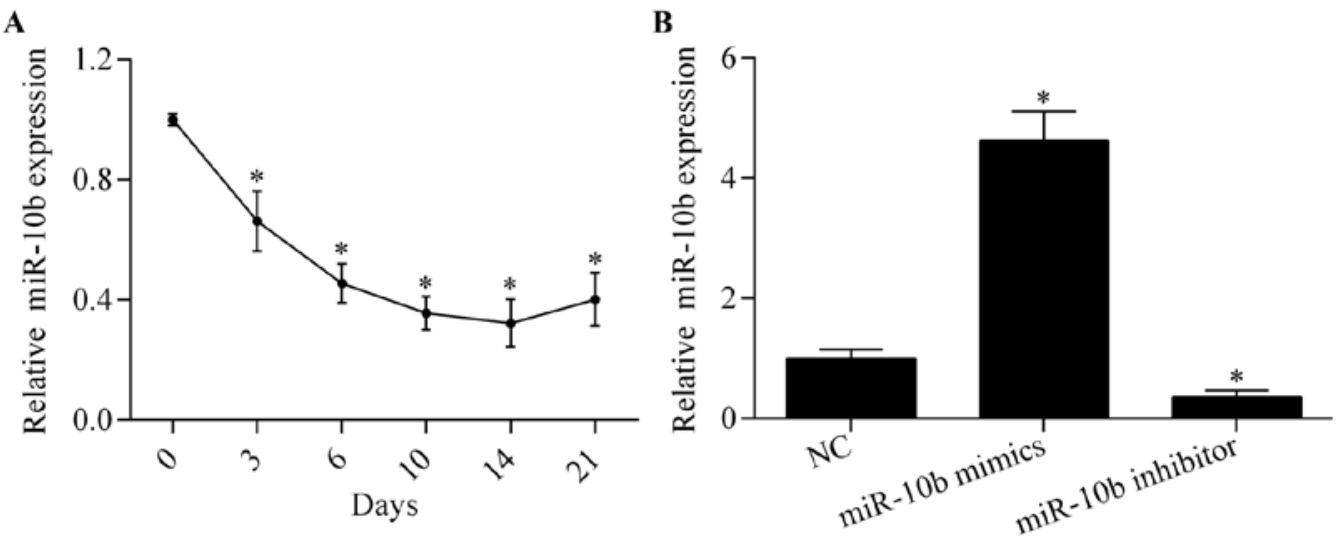

Figure 1. Expression of miR-10b during osteoblast differentiation. (A) RT-qPCR analysis of miR-10b expression in MC3T3-E1 cells post-osteoblast differentiation induction. " $\mathrm{p}<0.05$ vs. day 0 . (B) The expression of miR-10b in MC3T3-E1 cells transfected with miR-10b mimics or miR-10b inhibitor was detected by RT-qPCR after transfection of $24 \mathrm{~h}$. " $\mathrm{p}<0.05$ vs. NC.

at $4^{\circ} \mathrm{C}$. The cell sediments were treated with buffer A containing $1 \mathrm{mM}$ pheylmethylsulfonyl fluoride (PMSF) and incubated in an ice bath for $10 \mathrm{~min}$. Afterward, buffer $\mathrm{B}$ was added and incubated for $1 \mathrm{~min}$ followed by centrifugation at $12,000 \mathrm{x} \mathrm{g}$ for $15 \mathrm{~min}$ at $4^{\circ} \mathrm{C}$. The supernatants containing cytoplasmic fractions were collected. The sediments were collected and re-suspended in nuclear protein extraction agent and subjected to an ice bath for $30 \mathrm{~min}$ with vortexing at an interval of $2 \mathrm{~min}$. After centrifugation $\left(12,000 \mathrm{x}\right.$ g for $15 \mathrm{~min}$ at $\left.4^{\circ} \mathrm{C}\right)$, the supernatants containing nuclear protein was collected. Protein concentration was measured by a BCA kit (Beyotime). Equal amounts of proteins were loaded on $10 \%$ sodium dodecyl sulfate polyacrylamide gels for separation. The separated proteins were electro-blotted to a polyvinylidene fluoride membrane (Millipore, Boston, MA, USA). The membrane was blocked with $3 \%$ non-fat milk in Tris-buffered saline containing $0.1 \%$ Tween-20 (TBST) for $1 \mathrm{~h}$ at $37^{\circ} \mathrm{C}$. Then, the membrane was blotted with primary antibodies at appropriate dilutions at $4^{\circ} \mathrm{C}$ overnight. After washes with TBST, the membrane was incubated with horseradish peroxidase-conjugated secondary antibodies (1:2,000; goat anti-rabbit IgG; sc-2004; Santa Cruz Biotechnology, Inc.) for $1 \mathrm{~h}$ at $37^{\circ} \mathrm{C}$. The protein signals were visualized using Pierce ECL Western Blotting kit (Pierce, Rockford, IL, USA). Quantitative analysis of the protein bands was performed by Image-Pro Plus 6.0 software (Media Cybernetics, Inc., Rockville, MD, USA). The primary antibodies including anti-Bcl6 (sc-368), anti-STAT1 (sc-346), anti-Runx2 (sc-10758), anti-GAPDH (sc-25778) and anti-Lamin B (sc-6217) were purchased from Santa Cruz Biotechnology, Inc.

Data analysis. All data are presented as means \pm standard deviation. The statistical analysis was performed by SPSS version 18.0 (SPSS Inc., Chicago, IL, USA). Differences were assessed by one-way analysis of variance followed by a Bonferroni correction. A p-value of $<0.05$ was regarded as indicative of statistically significance.

\section{Results}

miR-10b is downregulated during osteoblast differentiation. To investigate the potential role of miR-10b in osteoblast differentiation, we examined the expression pattern of miR-10b during osteoblast differentiation in MC3T3-E1 cells by RT-qPCR. The results showed that miR-10b was significantly downregulated post-osteoblast differentiation (Fig. 1A), indicating a critical role of miR-10b involved in osteoblast differentiation.

miR $-10 b$ regulates osteoblast differentiation. To explore the exact biological effect of miR-10b on osteoblast differentiation, miR-10b was overexpressed or suppressed by transfecting miR-10b mimics or miR-10b inhibitors, respectively (Fig. 1B). We then examined the effect of miR-10b overexpression or suppression on osteoblast differentiation by evaluating ALP activity and matrix mineralization. The results showed that both ALP activity (Fig. 2A) and matrix mineralization (Fig. 2B) were markedly repressed by miR-10b overexpression. Conversely, the suppression of miR-10b significantly promoted ALP activity (Fig. 2A) and matrix mineralization (Fig. 2B). Moreover, we detected the expression of osteoclast marker genes, including ALP, COL1A1 and OPN by RT-qPCR. We found that the expression of these genes was significantly suppressed by miR-10b overexpression, while miR-10b suppression markedly elevated the expression of these genes (Fig. 3). Overall, these results suggest that miR-10b suppression promotes osteoblast differentiation.

Bcl6 is a target gene of miR-10b in osteoclasts. To investigate the underlying mechanism by which $\mathrm{miR}-10 \mathrm{~b}$ regulates osteoblast differentiation, we predicted the potential target genes of miR-10b by bioinformatics analysis. Among these putative target genes, Bcl6, a critical regulator of osteoblast differentiation (21), gained our interest for further analysis. The complementary seed-matched wild-type or mutant-type binding sites between miR-10b and Bcl6 3'-UTR are described in Fig. 4A. To verify the interaction between miR-10b and Bcl6 3'-UTR, wild-type or mutant-type pmirGLO-Bcl6 3'-UTR was co-transfected into MC3T3-E1 cells with miR-10b mimics or miR-10b inhibitor. The results showed that miR-10b overexpression significantly inhibited the luciferase reporter activity of wild-type luciferase vector while miR-10b suppression increased the luciferase reporter activity (Fig. 4B). However, 

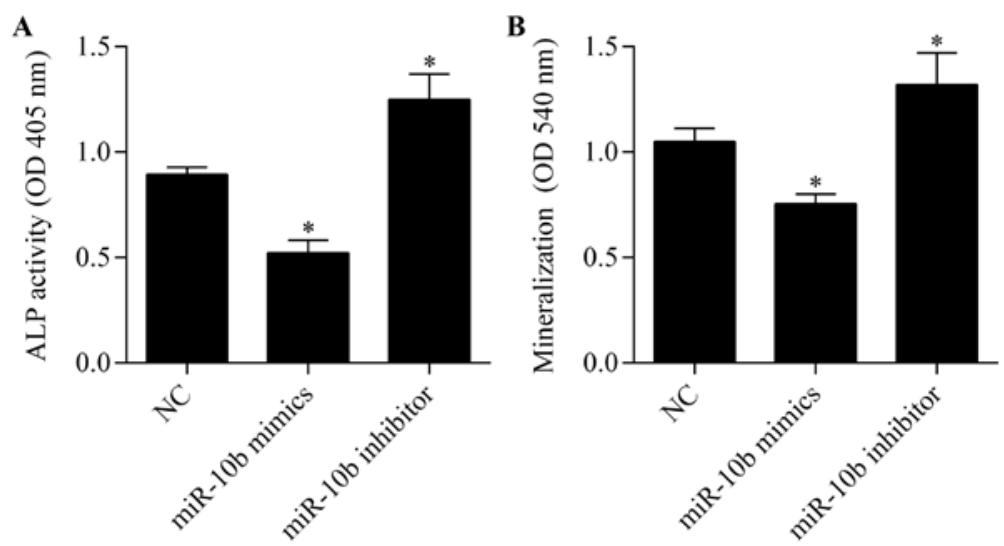

Figure 2. miR-10b regulates osteoblast differentiation. Cells were transfected with miR-10b mimics or inhibitor for $24 \mathrm{~h}$ and then cultured in differentiation medium for inducing osteoblast differentiation for 6 days. (A) Alkaline phosphatase (ALP) activity was detected by an ALP activity assay kit and the absorbance was measured at $405 \mathrm{~nm}$. (B) Matrix mineralization was determined by Alizarin Red S staining and the absorbance was measured at $540 \mathrm{~nm}$. " $\mathrm{p}<0.05 \mathrm{vs}$. NC.
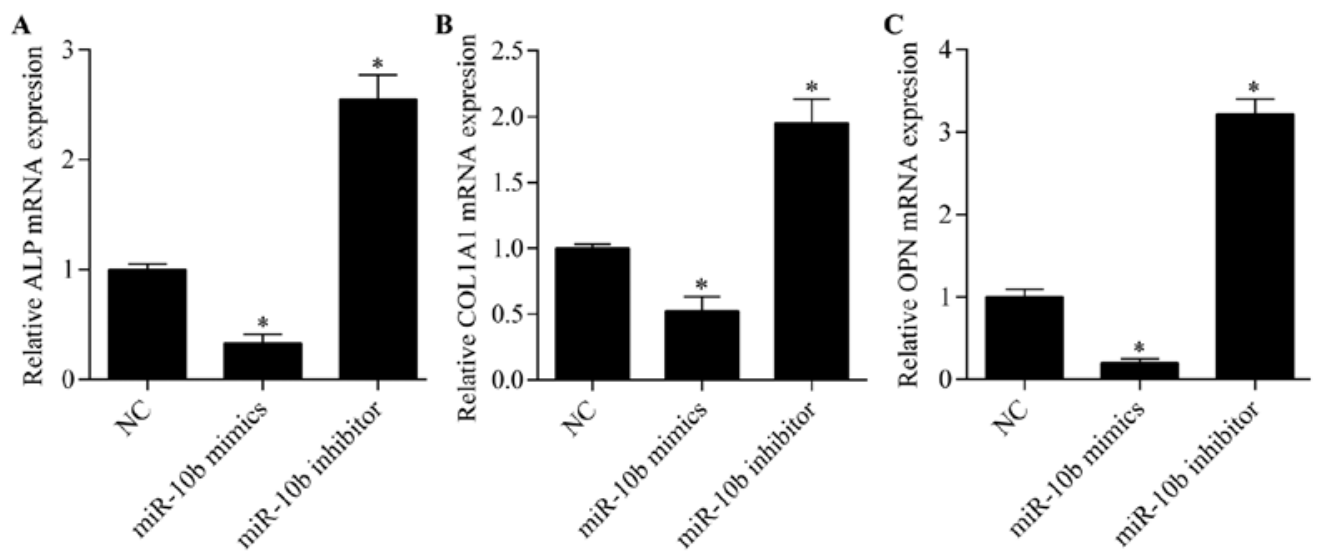

Figure 3. miR-10b affects the expression of osteoclast marker genes. The mRNA expression of (A) alkaline phosphatase (ALP), (B) collagen type I 1 (COL1A1) and (C) osteopontin (OPN) was detected by RT-qPCR analysis in MC3T3-E1 cells. Cells were transfected with miR-10b mimics or inhibitor for $24 \mathrm{~h}$ and then cultured in differentiation medium to induce osteoblast differentiation for 6 days. ${ }^{*} \mathrm{p}<0.05$ vs. NC.

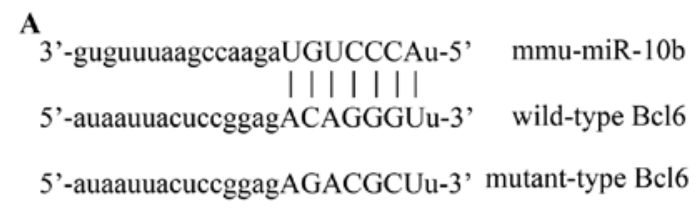

B
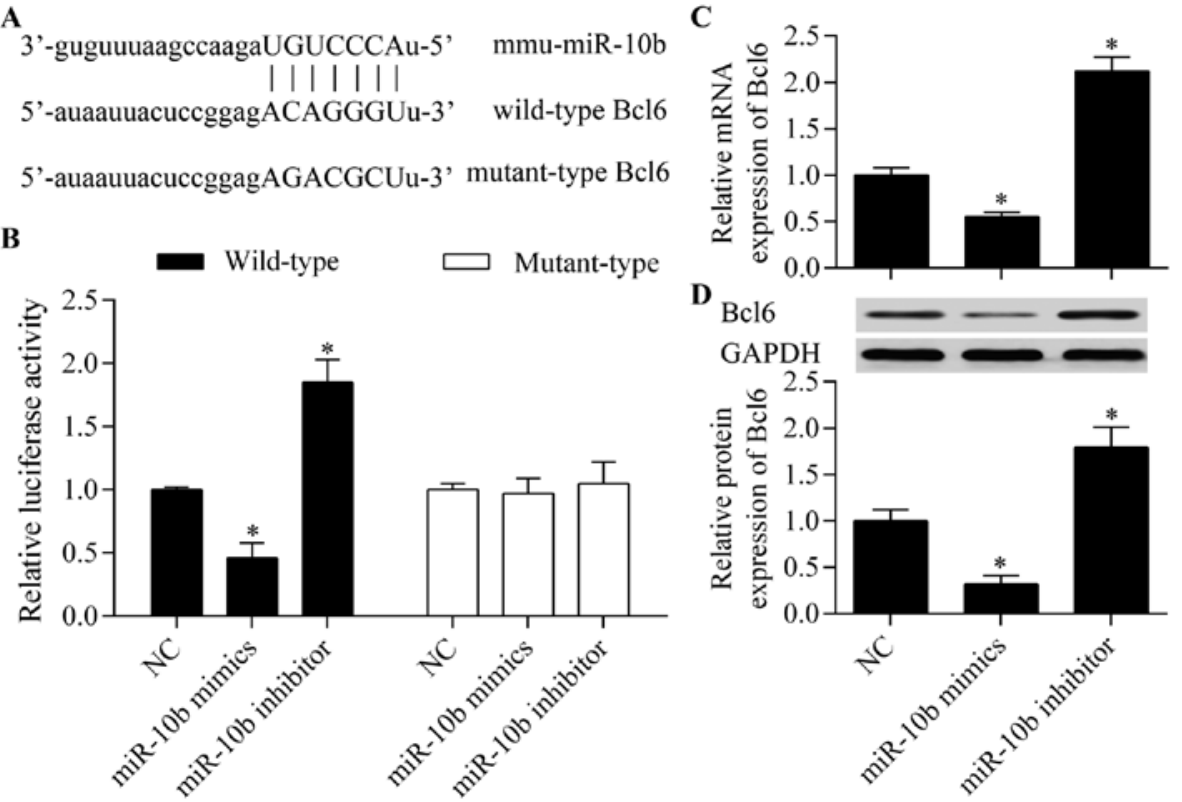

Figure 4. B cell lymphoma 6 (Bcl6) is a target gene of miR-10b. (A) Sequence alignment of seed-matched binding sites between mouse miR-10b with Bcl6 3'-UTR. (B) The effect of miR-10b on luciferase activity in MC3T3-E1 cells transfected with the Bcl6 3'-UTR luciferase reporter. The luciferase activity was detected by dual-luciferase method after a 48 -h transfection. * $\mathrm{p}<0.05$ vs. NC. (C) mRNA and (D) protein expression of Bcl6 were detected by RT-qPCR or western blot analysis, respectively. MC3T3-E1 cells were transfected with miR-10b mimics or miR-10b inhibitors for 24 h. * $\mathrm{p}<0.05$ vs. NC. 
A

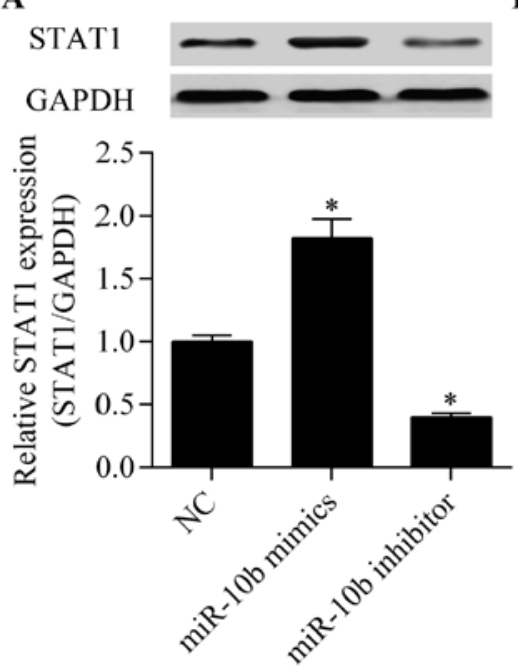

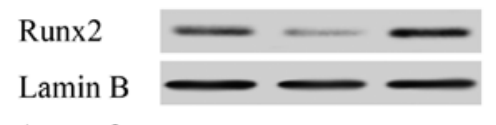

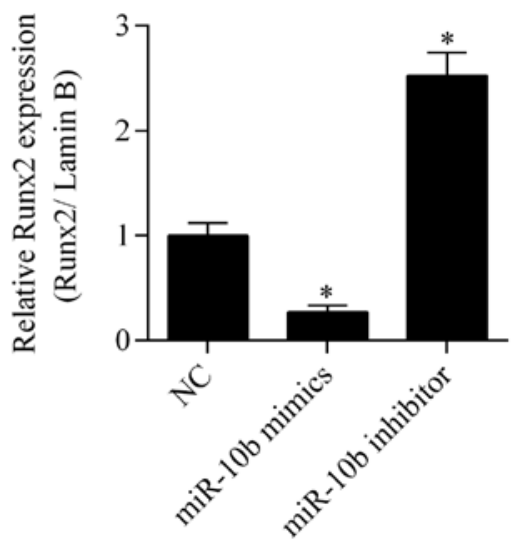

Figure 5. miR-10b regulates signal transducer and activator of transcription 1 (STAT1)/Runt-related transcription factor 2 (Runx2) signaling. MC3T3-E1 cells were transfected with miR-10b mimics or miR-10b inhibitors for $24 \mathrm{~h}$, followed by osteoblast differentiation for 6 days. (A) Western blot analysis of STAT1 protein expression. Glyceraldehyde 3-phosphate dehydrogenase (GAPDH) was used for normalization. (B) Western blot analysis of Runx2 protein expression in nuclear fractions. Lamin B was used for normalization. ${ }^{*} \mathrm{p}<0.05 \mathrm{vs}$. NC.

A

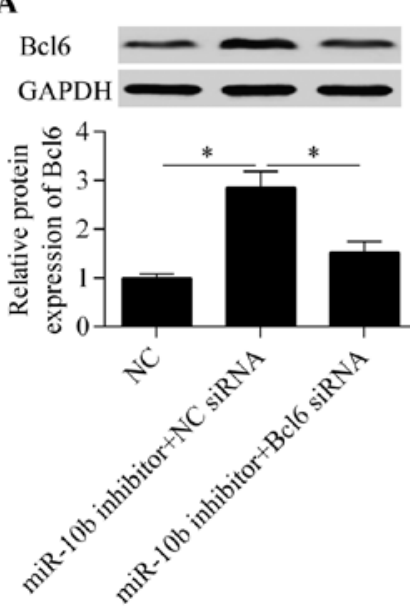

B

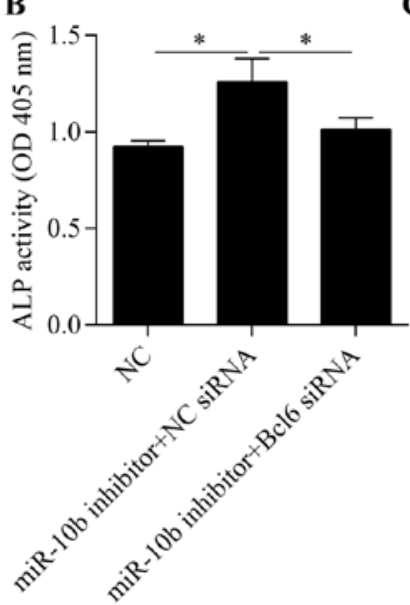

C

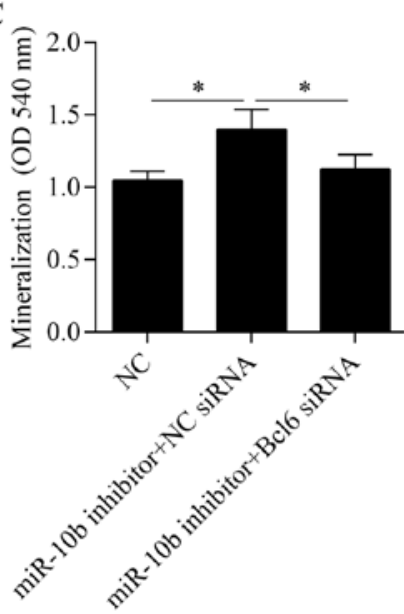

Figure 6. B cell lymphoma 6 (Bc16) knockdown abrogates the effects of miR-10b suppression on osteoblast differentiation. (A) Western blot analysis of Bcl6 protein expression in MC3T3-E1 cells co-transfected with miR-10b inhibitor and Bcl6 siRNA for $24 \mathrm{~h}$. After the induction of osteoblast differentiation for 6 days, (B) alkaline phosphatase (ALP) activity and (C) mineralization were detected. "p $<0.05$.

no obvious effect of miR-10b overexpression or suppression on mutant-type luciferase vector was observed (Fig. 4B). These data indicated that miR-10b directly targeted the 3'-UTR of Bcl6. To further confirm that this interaction is effective, we then examined the effect of miR-10b on Bcl6 expression. The results showed that miR-10b significantly suppressed the mRNA (Fig. 4C) and protein (Fig. 4D) expression of Bcl6, whereas miR-10b suppression increased Bcl6 expression. Taken together, these results suggest that Bcl6 is the target of miR-10b in osteoblasts.

miR $-10 b$ regulates STAT1/Runx2 signaling. Considering the regulatory effect of miR-10b on Bcl6 expression, we detected the effect of miR-10b on downstream target genes of Bcl6 involved in osteoblast differentiation. STAT1, which is an important regulator for osteoblast differentiation (21), has been reported as a target gene of Bcl6 (21). STAT1 negatively regulates osteoblast differentiation by repressing Runx2 nuclear translocation (18). We found that the overexpression of miR-10b increased while the suppression of miR-10b decreased STAT1 expression (Fig. 5A). Moreover, the Runx2 nuclear translocation was significantly blocked by miR-10b overexpression whereas miR-10b suppression promoted Runx2 nuclear translocation (Fig. 5B). These results indicate that miR-10b affects STAT1/Runx2 signaling.

miR-10b regulates osteoblast differentiation through targeting $B c l 6$. To confirm that the regulatory effect of miR-10b on osteoblast differentiation is regulated by targeting Bcl6, we silenced Bcl6 expression along with miR-10b suppression. The results showed that the promotive effect of miR-10b suppression on Bcl6 expression was significantly blocked by 
A

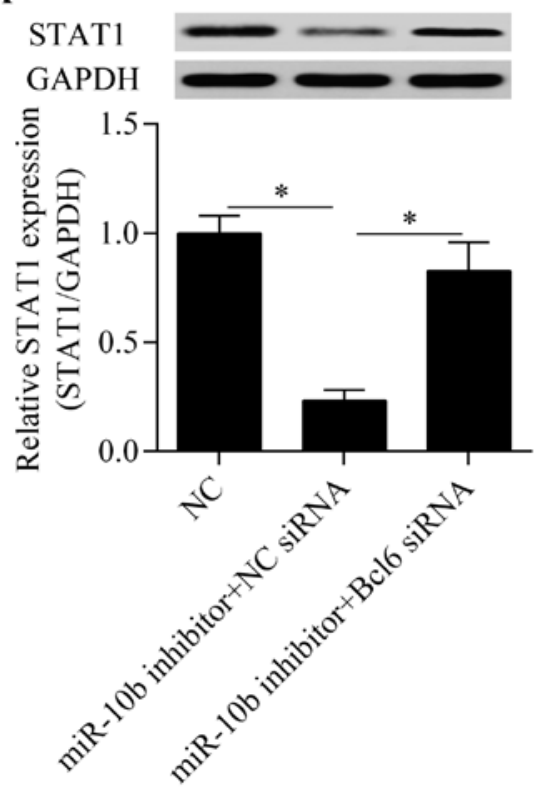

B

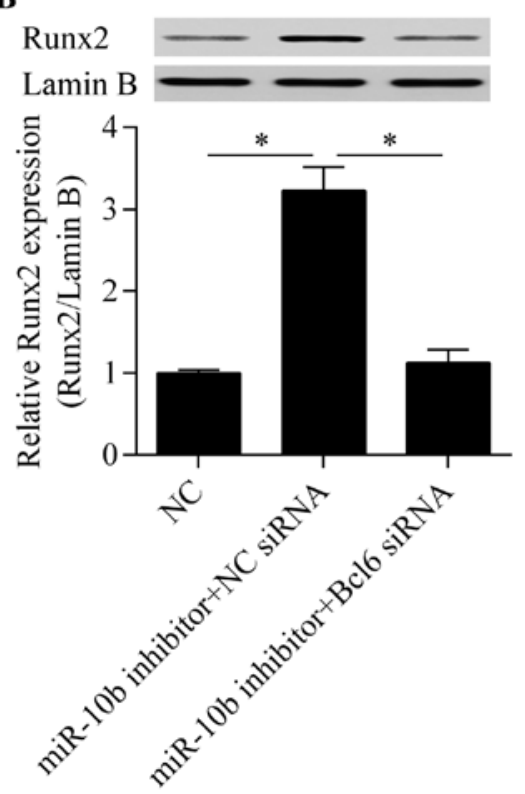

Figure 7. B cell lymphoma 6 (Bcl6) knockdown reverses the effects of miR-10b suppression on signal transducer and activator of transcription 1 (STAT1)/Runt-related transcription factor 2 (Runx2) signaling. MC3T3-E1 were cells co-transfected with miR-10b inhibitor and Bcl6 siRNA for $24 \mathrm{~h}$, followed by osteoblast differentiation for 6 days. (A) Western blot analysis of STAT1 protein expression. Glyceraldehyde 3-phosphate dehydrogenase (GAPDH) was used for normalization. (B) Western blot analysis of Runx2 protein expression in nuclear fractions. Lamin B was used for normalization. " $\mathrm{p}<0.05$.

Bc16 knockdown (Fig. 6A). As expected, the osteoblast differentiation promoted by miR-10b suppression was apparently abolished by Bcl6 knockdown (Fig. 6B and C). Moreover, the miR-10b suppression-induced inhibitory effect on STAT1 expression (Fig. 7A) and the promotive effect on Runx2 nuclear translocation (Fig. 7B) were significantly reversed by Bcl6 knockdown.

\section{Discussion}

A growing body of evidence has highlighted the critical role of miRNAs in bone homeostasis (28). Osteoblasts secrete ALP and bone matrix proteins to promote bone formation (4). Through targeting critical genes involved in osteoblast differentiation, miRNAs regulate osteoblast differentiation (9-12). Targeting osteoblast differentiation by miRNAs has become a promising therapeutic strategy for inhibiting bone loss. In this study, we showed that miR-10b is a novel miRNA involved in regulating osteoblast differentiation. We delineated that miR-10b regulates osteoblast differentiation through targeting Bcl6, implying an important role of miR-10b in bone homeostasis.

miR-10b has been widely studied in cancer $(29,30)$, angiogenesis (31) and embryonic development (32) by focusing on different targets. It has been found that miR-10b regulates myeloid differentiation and neuroblastoma cell differentiation $(24,25)$. Okamoto et al found that miR-10b was significantly downregulated during osteoblast differentiation (26). In line with these findings, our results also showed decreased miR-10b expression during osteoblast differentiation. Functional experiments demonstrated that the overexpression of miR-10b suppressed osteoblast differentiation while the suppression of miR-10b promoted osteoblast differentiation. Our results suggest that miR-10b is an osteoblast differentiation-related
miRNA. However, the underlying mechanism needs to be investigated.

To investigate the underlying mechanism by which miR-10b regulates osteoblast differentiation, we aimed to identify the functional target of miR-10b. Through bioinformatics analysis, we found that Bcl6 is a putative target gene of miR-10b. Bcl6 has been reported to positively regulate osteoblast differentiation (21). Bcl6 is a transcriptional repressor primarily expressed in B lymphocytes and regulates B lymphocyte growth and development $(22,23)$. Bcl6 participates in the regulation of B-cell lymphomas and numerous types of human cancer $(33,34)$. Bcl6 also regulates $\mathrm{T}$ follicular helper cell differentiation $(35,36)$ and germinal center formation $(37,38)$. A previous study showed that Bcl6 inhibits osteoclast differentiation (39). Bcl6 suppresses the expression of nuclear factor of activated T cells $\mathrm{cl}$ (NFATc1) which promotes osteoclast differentiation (40-42). Importantly, Bcl6 also participates in osteoblast differentiation (21). It has been reported that Bcl6 promotes osteoblast differentiation through the transcriptional repression of STAT1 (21). STAT1 is a negative regulator of osteoblast differentiation (18-20). The lack of STAT1 promotes bone formation and bone mass (18). STAT1 inhibits osteoblast differentiation by blocking Runx2 nuclear translocation (18). Targeting the inhibition of STAT1 by various agents showed a promotive effect on osteoblast differentiation (43-46). In this study, we demonstrated that inhibition of STAT1 by miR-10b suppression-induced Bcl6 promoted Runx2 nuclear translocation and osteoblast differentiation, indicating a potential strategy for the control of osteoblast differentiation by targeting STAT1.

Several studies have reported that Bcl6 is targeted by various miRNAs $(47,48)$. Bcl6 has been reported to be targeted by miR-155 in macrophages involved in atherosclerosis (49). miR-127 regulates breast cancer cell proliferation 
and senescence by targeting Bcl6 (50). miR-187 suppresses lung cancer development by targeting Bcl6 (51). Consistently, the targeting of Bcl6 by miR-187 also functions in regulating diffuse large B-cell lymphoma cell apoptosis (52). Here, our study for the first time reported that miR-10b is a novel miRNA that targets and modulates Bcl6 expression in osteoblasts. Taken together, these findings suggest that Bcl6 undergoes epigenetic regulation in various cell types and pathological processes.

Overall, this study showed that miR-10b participates in osteoblast differentiation by targeting Bcl6 and STAT1/Runx2 signaling. Our findings provide novel insight into understanding the molecular mechanism of osteoblast differentiation. miR-10b has great potential to serve as an effective target for bone formation.

\section{References}

1. Franceschi RT: The developmental control of osteoblast-specific gene expression: Role of specific transcription factors and the extracellular matrix environment. Crit Rev Oral Biol Med 10: 40-57, 1999.

2. Park H, Noh AL, Kang JH, Sim JS, Lee DS and Yim M: Peroxiredoxin II negatively regulates lipopolysaccharideinduced osteoclast formation and bone loss via JNK and STAT3. Antioxid Redox Signal 22: 63-77, 2015.

3. Diddle AW and Smith IQ: Postmenopausal osteoporosis: The role of estrogens. South Med J 77: 868-874, 1984.

4. Canalis E, Economides AN and Gazzerro E: Bone morphogenetic proteins, their antagonists, and the skeleton. Endocr Rev 24: 218-235, 2003.

5. Huang C, Geng J and Jiang S: MicroRNAs in regulation of osteogenic differentiation of mesenchymal stem cells. Cell Tissue Res: Jul 18, 2016 (Epub ahead of print).

6. Bartel DP: MicroRNAs: Genomics, biogenesis, mechanism, and function. Cell 116: 281-297, 2004.

7. Ambros V: The functions of animal microRNAs. Nature 431: 350-355, 2004.

8. Bartel DP: MicroRNAs: Target recognition and regulatory functions. Cell 136: 215-233, 2009.

9. Vishal M, Vimalraj S, Ajeetha R, Gokulnath M, Keerthana R, He Z, Partridge NC and Selvamurugan N: MicroRNA-590-5p stabilizes Runx 2 by targeting Smad7 during osteoblast differentiation. J Cell Physiol 232: 371-380, 2017.

10. Yan J, Guo D, Yang S, Sun H, Wu B and Zhou D: Inhibition of miR-222-3p activity promoted osteogenic differentiation of hBMSCs by regulating Smad5-RUNX2 signal axis. Biochem Biophys Res Commun 470: 498-503, 2016.

11. Hu Z, Wang Y, Sun Z, Wang H, Zhou H, Zhang L, Zhang S and Cao X: miRNA-132-3p inhibits osteoblast differentiation by targeting Ep300 in simulated microgravity. Sci Rep 5: 18655, 2015.

12. Fukuda T, Ochi H, Sunamura S, Haiden A, Bando W, Inose H, Okawa A, Asou Y and Takeda S: MicroRNA-145 regulates osteoblastic differentiation by targeting the transcription factor Cbfb. FEBS Lett 589: 3302-3308, 2015.

13. Chen D, Zhao M and Mundy GR: Bone morphogenetic proteins. Growth Factors 22: 233-241, 2004.

14. Komori T, Yagi H, Nomura S, Yamaguchi A, Sasaki K, Deguchi K, Shimizu Y, Bronson RT, Gao YH, Inada M, et al: Targeted disruption of Cbfal results in a complete lack of bone formation owing to maturational arrest of osteoblasts. Cell 89 : 755-764, 1997

15. Ducy P, Zhang R, Geoffroy V, Ridall AL and Karsenty G: Osf2/Cbfa1: A transcriptional activator of osteoblast differentiation. Cell 89: 747-754, 1997.

16. Nakashima K, Zhou X, Kunkel G, Zhang Z, Deng JM, Behringer RR and de Crombrugghe $B$ : The novel zinc fingercontaining transcription factor osterix is required for osteoblast differentiation and bone formation. Cell 108: 17-29, 2002

17. Takayanagi H, Kim S, Koga T and Taniguchi T: Stat1-mediated cytoplasmic attenuation in osteoimmunology. J Cell Biochem 94 232-240, 2005
18. Kim S, Koga T, Isobe M, Kern BE, Yokochi T, Chin YE, Karsenty G, Taniguchi T and Takayanagi H: Statl functions as a cytoplasmic attenuator of Runx2 in the transcriptional program of osteoblast differentiation. Genes Dev 17: 1979-1991, 2003

19. Takayanagi H, Ogasawara K, Hida S, Chiba T, Murata S, Sato K Takaoka A, Yokochi T, Oda H, Tanaka K, et al: T-cell-mediated regulation of osteoclastogenesis by signalling cross-talk between RANKL and IFN-gamma. Nature 408: 600-605, 2000.

20. Takayanagi H, Kim S, Matsuo K, Suzuki H, Suzuki T, Sato K, Yokochi T, Oda H, Nakamura K, Ida N, et al: RANKL maintains bone homeostasis through c-Fos-dependent induction of interferon-beta. Nature 416: 744-749, 2002.

21. Fujie A, Funayama A, Miyauchi Y, Sato Y, Kobayashi T, Kanagawa H, Katsuyama E, Hao W, Tando T, Watanabe R, et al: Bcl6 promotes osteoblastogenesis through Stat1 inhibition. Biochem Biophys Res Commun 457: 451-456, 2015.

22. Chang CC, Ye BH, Chaganti RS and Dalla-Favera R: BCL-6, a $\mathrm{POZ/zinc-finger} \mathrm{protein,} \mathrm{is} \mathrm{a} \mathrm{sequence-specific} \mathrm{transcriptional}$ repressor. Proc Natl Acad Sci USA 93: 6947-6952, 1996.

23. Jardin F, Ruminy P, Bastard C and Tilly H: The BCL6 protooncogene: A leading role during germinal center development and lymphomagenesis. Pathol Biol (Paris) 55: 73-83, 2007.

24. Zou Q, Tan S, Yang Z, Wang J, Xian J, Zhang S, Jin H, Yang L, Wang L and Zhang L: The human nucleophosmin 1 mutation $\mathrm{A}$ inhibits myeloid differentiation of leukemia cells by modulating miR-10b. Oncotarget 7: 71477-71490, 2016.

25. Foley NH, Bray I, Watters KM, Das S, Bryan K, Bernas T, Prehn JH and Stallings RL: MicroRNAs 10a and 10b are potent inducers of neuroblastoma cell differentiation through targeting of nuclear receptor corepressor 2. Cell Death Differ 18: 1089-1098, 2011.

26. Okamoto H, Matsumi Y, Hoshikawa Y, Takubo K, Ryoke K and Shiota G: Involvement of microRNAs in regulation of osteoblastic differentiation in mouse induced pluripotent stem cells. PLoS One 7: e43800, 2012.

27. Hassan MQ, Maeda Y, Taipaleenmaki H, Zhang W, Jafferji M, Gordon JA, Li Z, Croce CM, van Wijnen AJ, Stein JL, et al: miR-218 directs a Wnt signaling circuit to promote differentiation of osteoblasts and osteomimicry of metastatic cancer cells. J Biol Chem 287: 42084-42092, 2012.

28. Boyce BF, Rosenberg E, de Papp AE and Duong LT: The osteoclast, bone remodelling and treatment of metabolic bone disease. Eur J Clin Invest 42: 1332-1341, 2012.

29. Wang J, Wang B, Chen LQ, Yang J, Gong ZQ, Zhao XL, Zhang CQ and Du KL: miR-10b promotes invasion by targeting KLF4 in osteosarcoma cells. Biomed Pharmacother 84: 947-953, 2016.

30. Knirsh R, Ben-Dror I, Modai S, Shomron N and Vardimon L: MicroRNA $10 \mathrm{~b}$ promotes abnormal expression of the protooncogene c-Jun in metastatic breast cancer cells. Oncotarget 7: 59932-59944, 2016.

31. Wang X, Ling CC, Li L, Qin Y, Qi J, Liu X, You B, Shi Y, Zhang J, Jiang Q, et al: MicroRNA-10a/10b represses a novel target gene mibl to regulate angiogenesis. Cardiovasc Res 110: 140-150, 2016.

32. Giusti J, Pinhal D, Moxon S, Campos CL, Münsterberg A and Martins C: MicroRNA-10 modulates Hox genes expression during Nile tilapia embryonic development. Mech Dev 140: $12-18,2016$

33. Duan S, Cermak L, Pagan JK, Rossi M, Martinengo C, di Celle PF, Chapuy B, Shipp M, Chiarle R and Pagano M: FBXO11 targets BCL6 for degradation and is inactivated in diffuse large B-cell lymphomas. Nature 481: 90-93, 2012.

34. Wu Q, Liu X, Yan H, He YH, Ye S, Cheng XW, Zhu GL, Wu WY, Wang XN, Kong XJ, et al: B-cell lymphoma 6 protein stimulates oncogenicity of human breast cancer cells. BMC Cancer 14: 418, 2014.

35. Nance JP, Bélanger S, Johnston RJ, Hu JK, Takemori T and Crotty S: Bcl6 middle domain repressor function is required for $\mathrm{T}$ follicular helper cell differentiation and utilizes the corepressor MTA3. Proc Natl Acad Sci USA 112: 13324-13329, 2015.

36. Liu X, Lu H, Chen T, Nallaparaju KC, Yan X, Tanaka S, Ichiyama $\mathrm{K}$, Zhang X, Zhang L, Wen X, et al: Genome-wide analysis identifies Bcl6-controlled regulatory networks during $\mathrm{T}$ follicular helper cell differentiation. Cell Rep 14: 1735-1747, 2016.

37. Hu G and Zhao K: Looping around Bcl6 in germinal center to sharpen B cell immunity. Immunity 45: 459-461, 2016. 
38. Ying Z, Mei M, Zhang P, Liu C, He H, Gao F and Bao S: Histone arginine methylation by PRMT7 controls germinal center formation via regulating Bcl6 transcription. J Immunol 195: $1538-1547,2015$

39. Miyauchi Y, Ninomiya K, Miyamoto H, Sakamoto A, Iwasaki R, Hoshi H, Miyamoto K, Hao W, Yoshida S, Morioka H, et al: The Blimp1-Bcl6 axis is critical to regulate osteoclast differentiation and bone homeostasis. J Exp Med 207: 751-762, 2010.

40. Park-Min KH, Lee EY, Moskowitz NK, Lim E, Lee SK, Lorenzo JA, Huang C, Melnick AM, Purdue PE, Goldring SR, et al: Negative regulation of osteoclast precursor differentiation by $\mathrm{CD} 11 \mathrm{~b}$ and $\beta 2$ integrin-B-cell lymphoma 6 signaling. J Bone Miner Res 28: 135-149, 2013

41. Swarnkar G, Shim K, Nasir AM, Seehra K, Chen HP, Mbalaviele G and Abu-Amer Y: Myeloid deletion of nemo causes osteopetrosis in mice owing to upregulation of transcriptional repressors. Sci Rep 6: 29896, 2016.

42. Morita M, Yoshida S, Iwasaki R, Yasui T, Sato Y, Kobayashi T, Watanabe R, Oike T, Miyamoto K, Takami M, et al: Smad4 is required to inhibit osteoclastogenesis and maintain bone mass Sci Rep 6: 35221, 2016.

43. Li J, He X, Wei W and Zhou X: MicroRNA-194 promotes osteoblast differentiation via downregulating STAT1. Biochem Biophys Res Commun 460: 482-488, 2015.

44. Xiao WZ, Gu XC, Hu B, Liu XW, Zi Y and Li M: Role of microRNA-129-5p in osteoblast differentiation from bone marrow mesenchymal stem cells. Cell Mol Biol (Noisy-le-grand) 62: 95-99, 2016.

45. Tajima K, Takaishi H, Takito J, Tohmonda T, Yoda M, Ota N, Kosaki N, Matsumoto M, Ikegami H, Nakamura T, et al: Inhibition of STAT1 accelerates bone fracture healing. J Orthop Res 28: 937-941, 2010.
46. Yoshida K, Okamura H, Amorim BR, Hinode D, Yoshida H and Haneji T: PKR-mediated degradation of STAT1 regulates osteoblast differentiation. Exp Cell Res 315: 2105-2114, 2009.

47. Tryndyak VP, Ross SA, Beland FA and Pogribny IP Downregulation of the microRNAs miR-34a, miR-127, and miR-200b in rat liver during hepatocarcinogenesis induced by a methyl-deficient diet. Mol Carcinog 48: 479-487, 2009.

48. Martín-Pérez D, Vargiu P, Montes-Moreno S, León EA, Rodríguez-Pinilla SM, Lisio LD, Martínez N, Rodríguez R, Mollejo M, Castellvi J, et al: Epstein-Barr virus microRNAs repress BCL6 expression in diffuse large B-cell lymphoma. Leukemia 26: 180-183, 2012.

49. Nazari-Jahantigh M, Wei Y, Noels H, Akhtar S, Zhou Z, Koenen RR, Heyll K, Gremse F, Kiessling F, Grommes J, et al: MicroRNA-155 promotes atherosclerosis by repressing Bcl6 in macrophages. J Clin Invest 122: 4190-4202, 2012.

50. Chen J, Wang M, Guo M, Xie Y and Cong YS: miR-127 regulates cell proliferation and senescence by targeting BCL6. PLoS One 8: e80266, 2013.

51. Sun C, Li S, Yang C, Xi Y, Wang L, Zhang F and Li D: MicroRNA187-3p mitigates non-small cell lung cancer (NSCLC) development through Downregulation of BCL6. Biochem Biophys Res Commun 471: 82-88, 2016.

52. Huang F, Jin Y and Wei Y: MicroRNA-187 induces diffuse large B-cell lymphoma cell apoptosis via targeting BCL6. Oncol Lett 11: 2845-2850, 2016. 